DOI: $10.47460 /$ uct.v24i104.362

\title{
ACTIVIDAD FÚNGICA DE ASPERGILLUS SPP. MEDIANTE LA APLICACIÓN DE AZADIRACHTA INDICO Y EUCALIPTUS SPP
}

\author{
Hurtado Erika ${ }^{1}$, Ballestero Francisco ${ }^{2}$, Banchón Carlos $^{3}$ \\ ehurtadoescobar@gmail.com ${ }^{1}$, andres.ballesteros@utelvt.edu.ec ${ }^{2}$ \\ cbanchon@uagraria.edu.ec ${ }^{3}$ \\ https://orcid.org/0000-0001-9178-2439 ${ }^{1}$ https://orcid.org/0000-0002-1862-380X ${ }^{2}$ \\ https://orcid.org/0000-0002-0388-19883 \\ Universidad de Barcelona ${ }^{1}$, Universidad Técnica de Esmeraldas Luis Vargas Torres ${ }^{2}$ \\ Universidad Agraria del Ecuador ${ }^{3}$ \\ Barcelona - España
}

Recibido (05/08/20), Aceptado (17/08/20)

\begin{abstract}
Resumen: Las continuas aplicaciones de fungicidas químicos en los cultivos a menudo son cada vez dañinas tanto para la salud de las personas y animales como para el ambiente. De acuerdo a esta problemática se realizó una investigación para evaluar el efecto de extractos naturales a partir de Azaridachta indico y Eucaliptus spp, frente a Aspergillus terreus como medida de protección ambiental. Se realizaron 5 tratamientos, (1) A. indico (2) Eucaliptus spp. (3) Mezcla de extractos (4) Testigo Control y (5) fungicida químico Skul. El fungicida químico tuvo 100\% de efectividad. Entre los extractos naturales la mezcla fue la más efectiva para inhibir el crecimiento de A. terreus. Siendo una alternativa agroecológica para cultivos con problemas fitosanitarios.
\end{abstract}

Palabras Clave: Aspergillus, eucalipto, neem, fungicidas químicos.

\section{FUNGAL ACTIVITY OF ASPERGILLUS SPP. THROUHG THE APLICATION OF AZADIRACHTA INDICA AND EUCALIPTUS SPP}

\begin{abstract}
The continuous application of chemical fungicides on crops is often increasingly, being harmful both to human and animal health and to the environment. According to this problem, an investigation was carried out to evaluate the effect of natural extracts from Azaridachta indico and Eucaliptus spp, against Aspergillus terreus as an environmental protection measure. Five treatments were performed, (1)A. indico (2)Eucalyptusspp.(3)Mixture ofextracts(4)ControlWitnessand(5)chemical fungicideSkul. The chemical fungicide was $100 \%$ effective. Among the natural extracts, the blend was the most effective in inhibiting the growth of A. terreus. Being an agroecological alternative for crops with phytosanitary problems.
\end{abstract}

Keywords: Aspergillus, eucalyptus, neem, chemical fungicides. 


\section{I.INTRODUCCIÓN}

Se considera que existen 6 millones de agroquímicos potencialmente tóxicos, en los que se emplean unos 100.000 componentes con efectos cancerígenos. La mayor parte de estos no contiene información sobre seguridad o riesgos asociados [1]. Los agroquímicos tienen la capacidad de generar contaminación en suelos y aguas tanto superficiales como subterráneas [2]. La agricultura, la actividad forestal y otros usos de la tierra generan el $21 \%$ del total mundial de emisiones anuales de gases de efecto invernadero [3]. En América Latina y el Caribe, el $37 \%$ de la tierra se utiliza para la agricultura y otro $47 \%$ está cubierto por bosques [4].En América Latina y el Caribe, casi el $1 \%$ del total de tierras agrícolas se dedica a la agricultura orgánica, un porcentaje superado solamente por Europa y Asia Central (1,4\%). América Latina cuenta con más que el doble del área bajo agricultura orgánica que América del Norte. La mayor parte de las tierras utilizadas para la agricultura orgánica en la región se encuentra en América del Sur. Además, República Dominicana y Uruguay tienen el mayor porcentaje de tierras agrícolas dedicadas a este sistema agrícola en la región (7,6\% y 6,5\%, respectivamente). Estos son los porcentajes más altos para la agricultura orgánica fuera de Europa. Argentina es el que sigue (2,2\%) [5]. Actualmente, hay más de 113 componentes activos inscritos como fungicidas comerciales a nivel mundial. Existe una gran cantidad de problemas contra el empleo eficaz de estos artículos en las zonas donde los hongos ha desarrollo resistencia. Por otra parte, se encuentra una intranquilidad internacional por exterminar el empleo de fungicidas químicos a nivel mundial.

Ecuador utiliza el 47,6\% de agroquímicos en las plantaciones. Las principales provincias son: Carchi $88 \%$, Imbabura 56,8\%, Esmeraldas 47,1\% y Sucumbíos 43,8 \% [6].En Ecuador, 81.248,36 hectáreas usan plaguicidas orgánicos, las cuales corresponden al 2,66\% de la superficie con cultivos transitorios y el 2,04\% cultivos permanentes, mientras para los plaguicidas químicos, la superficie de uso fue 1'764.426,44 de los cuales el $50,03 \%$ corresponde a cultivos permanentes y el $78,24 \%$ a cultivos transitorios. Dos de cada diez personas han recibido capacitación técnica sobre prevención, utilización y manejo de plaguicidas, el $82,66 \%$ corresponde al no y el 17,34\%, sí [7]. De los 1985 plaguicidas inscritos por Agrocalidad existen productos de clasificación extremada y altamente peligrosos. El reglamento ecuatoriano los restringe para administración aérea, sin embargo, el mismo estatuto admite expenderlos bajo disposición de un ingeniero agrónomo [8]. Los agroquímicos empleados para potenciar la productividad del suelo y cosechas inciden a diversas áreas tanto ambiental, como en la salud de las personas y animales [9]. En el país según los datos del informe "La equidad en la mira: la salud pública en Ecuador durante las últimas décadas" anuncia que la tasa de intoxicación por plaguicidas era de 0,8 por cada 100.000 habitantes en el año 1978, en el 2004 estos datos aumentaron a 15,2 presentándose 1.991 casos [8].Este proyecto tiene como meta formar una serie de conocimientos técnicos enlazados en la elaboración de un extracto vegetal amigable con el ambiente con fines antifúngicos. El estudio contribuirá a la carrera de Ingeniería Ambiental e Ingeniería Ecológica, creando microcosmos en ambiente controlado para el crecimiento de Aspergillus, con esto se establecerá un moderno sistema para una adecuada restauración ambiental. La obtención de fungicidas a partir de hojas Azadirachta indico y Eucaliptus son opciones para el tratamiento de hongos. Este sistema natural promueve nuevas metodologías y con esto cumple el objetivo de sustituir sustancias químicas nocivas con el ambiente por productos orgánicos y agroecológicos.

\section{II.DESARROLLO}

La utilización de extractos naturales como biofungicidas crean una alternativa a los fungicidas de origen químico y se emplean para disminuir el riesgo de enfermedades producidas por hongos en las plantas, dando lugar al aumento de la agricultura orgánica permitiendo contralar plagas: son menos amenazantes para el ambiente, disminuyen la contaminación de aguas y degradación del suelo, son biodegradables, económicos y accesibles para países poco desarrollados. Insecticidas, herbicidas y fungicidas también se aplican intensamente en muchos países, tanto desarrollados como en desarro1lo, lo que provoca la contaminación del agua dulce con compuestos carcinógenos y otros venenos que afectan al ser humano y a muchas formas de vida silvestre. Los plaguicidas también reducen la biodiversidad, ya que destruyen hierbas e insectos y con ellos las especies que sirven de alimento a pájaros y otros animales [4]. En Ecuador, Sangolquí se realizó un estudio en la Escuela Politécnica del Ejército sobre la eficiencia de extractos con principios activos de Eucalipto (Eucalyptus globulus), ajo (Allium sativum), crisantemo (Chrysanthemum cinerariaefolium) como fungicidas naturales para el control de Botrytis cinérea, Phragmidium mucronatum y Sphaerotheca pannosa presentes en el cultivo de rosas orgánicas [10]. Se recolectaron una serie de muestras como son: eucalipto, ajo y de crisantemo para la obtención de bioextractos y la recolección de muestras vegetales de rosas infectadas por hongos fitopatógenos. La etapa de laboratorio se basó en cuatro fases, el aislamiento de los hongos fitopatógenos Botrytis cinérea, 
Phragmidium mucronatum y Sphaerotheca pannosa, la obtención de extractos naturales a base de Eucalyptus globulus, Allium sativum, Chrysanthemum cinerariaefolium mediante tres métodos hidrodestilación, maceración con agua y alcohol 96\%, el análisis de extractos e identificación de los componentes mediante cromatografía de capa fina y la evaluación de la eficiencia fungicida in vitro de los extractos obtenidos, se ejecutó en un periodo de 8 meses.

Se realizó un diseño factorial de 9x2x4x2, teniendo nueve niveles para el factor uno: Extracto natural, dos niveles para el factor dos: diluyente $(\mathrm{v} / \mathrm{v})$, cuatro niveles para el factor tres: concentración (v/v) y dos niveles para el factor cuatro: tiempo de incubación (días/ horas); el fin de aplicar este diseño fue para investigar que tan eficientes son lo extractos vegetales como fungicidas naturales, los resultados se reflejan en una variable de respuesta (Y) que fueron los halos de inhibición medidos en $(\mathrm{mm})$ para Botrytis cinera y la proporción de conidios afectados con cambios morfológicos para Phragmidium mucronatum y Sphaerotheca pannos. El dieseño tuvo cinco repeticiones por tratamiento, dando un total de 144 tratamientos para cada hongo fitopatógeno. Es decir, se analizaron 96 muestras para Botrytis cinérea y 378 muestras para Phragmidium mucronatum y Sphaerotheca pannosa contando con los blancos para el control en cada tratamiento [10]. Los resultados obtenidos fueron sometidos al análisis de varianza ANOVA y pruebas de comparación de medias de Tukey al $0.05 \%$ utilizando el software estadístico STATGRAPHIC versión 2017. En conclusión, el rendimiento de obtención de los extractos de Eucalyptus globulus, Allium sativum y Chrysanthemum cinerariaefolium por maceración con agua y alcohol $96 \%$ fue alto, esto debido a factores tanto de tratamiento muestras vegetales como del proceso de concentración de extractos en rotavapor [10].

\section{III.METODOLOGÍA}

Se realizó un fungicida natural aplicado en especies de hongos aisladas (Aspergillus) en el laboratorio de microbiología de la Facultad de Ciencias Agrarias de la Universidad Agraria del Ecuador, campus Guayaquil. Los resultados sirvieron para inhibir el crecimiento de hongos a través extractos naturales a partir de A. indico y Eucaliptus. Fue una investigación exploratoria donde se aplicaron análisis cuantitativos y cualitativos sobre la actividad antifúngica de extractos naturales. La investigación inició desde la elaboración de los medios de cultivos para el hongo hasta el análisis final de los resultados. Se recopiló información de trabajos de investigación realizados anteriormente, donde se han obtenido resultados favorables para este estudio. Se empleó un diseño descriptivo ya que se observó el comportamiento del Aspergillus frente a la actividad antifúngica de los extractos naturales a partir de neem y eucalipto. Además de comparar la efectividad de los extractos naturales frente a un fungicida de origen químico. Se realizaron una serie de pruebas experimentales que permitieron aceptar o rechazar la hipótesis desarrollada. Se trabajó con variables independientes: tipo y dosis de tratamiento antifúngico a partir de extractos naturales , tipo y dosis de fungicida químico y tiempo de dosis/exposición, mientras que la variable dependiente fueron los tratamientos que se aplicaron.

Este proyecto estuvo constituido de los tratamientos siguientes:

T1. Dosis de extracto natural de neem (Azadirachta indico), T2. Dosis de extracto natural de eucalipto (Eucaliptus), T3. Mezcla de extracto natural de neem y eucalipto, T4. Control o testigo sin aplicación del fungicida, T5. Fungicida Químico (Skul).

\section{A.Diseño Experimental}

Se utilizaron $100 \mathrm{~g}$ de neem en $500 \mathrm{ml}$ de agua autoclavada con $0,9 \mathrm{~g}$ de sulfato monoácido (buffer). El proceso fue el mismo para eucalipto. Las hojas fueron cortadas en trozos iguales y lavadas con agua autoclavada. Se trituró continuamente ambos compuestos (agua y especie vegetal) a través del licuado.

Recursos Materiales: Hongo Aspergillus terreus, Hojas de Neem (A. indico), Hojas de Eucalipto (Eucalyptus spp.), Cloranfenicol, Sabouraud Dextrosa.

Materiales: Caja Petri, papel filtro, botellas, etanol al 96\%, cámara UV, incubadora, cámara, guantes, mascarillas, búffer

\section{B.Métodos}

Aspergillus terreus se encontraba aislado en cajas Petri, dentro del laboratorio de la Facultad de Ciencias Agrarias. Se preparó el medio de cultivo con Sabouraud Dextrosa aplicando la siguiente fórmula: $(65 \mathrm{~g} / 1000 \mathrm{ml})$. Se pesaron $26 \mathrm{~g}$ de Dextrosa Agar para cada $400 \mathrm{ml}$ de agua destilada. A esta solución se agregaron dos gotas de cloranfenicol y se procedió al autoclavado.

El medio de cultivo fue distribuido en cajas Petri dentro de la cámara de esterilización. Este medio fue esterilizado en UV durante un periodo de 20 minutos y guardado en refrigeración. En los tratamientos (1) Extracto natural del neem (A. indico), (2) Extracto natural del eucalipto (Eucaliptus), (3) Mezcla de neem + eucalipto y (5) Fungicida Químico (Skul) se subministró el respectivo fungicida. Se hizo un conteo del área de crecimiento de Aspergillus terreus, extracto que fue aplicado a través de discos de papel filtro con diámetros de 1 
cm durante un periodo de tiempo de 24, 48 y 72 horas. Estos tratamientos fueron comparados con el control o testigo para determinar la eficacia de los extractos frente al Aspergillus

\section{C.Elaboración de Fungicida Natural}

Se pesaron 100 gr de hojas de cada especie, estas fueron trituradas a través del licuado en solución de buffer. Se colocó la misma cantidad de extracto triturado y etanol al $96 \%$ en ultrasonido durante 30 min a $50 \mathrm{~W}$. Estos extractos fueron filtrados, colocados en botellas y refrigerados a $10{ }^{\circ} \mathrm{C}$. Una vez obtenido el bio- fungicida, se realizó un medio de cultivo con Sabouraud Dextrosa para la siembra de Aspergillus terreus. Se procedió a suministrar los respectivos tratamientos al hongo Aspergillus durante un periodo de 2 semanas en jornada diurna.

\section{D.Método de Análisis de Varianza}

Se realizaron 5 repeticiones por tratamiento. Tratamiento 1: Aplicación del bio-fungicida a base Neem. Se aplicó concentraciones controladas del bio fungicida. Tratamiento 2: Aplicación del bio-fungicida de Eucalipto: Se aplicó las respectivas dosis controladas de extracto natural. Luego, se procesaron los datos obtenidos para ser analizados estadísticamente los resultados sobre las variables dependientes. Tratamiento 3: Aplicación de mezcla de extracto natural de neem + eucalipto. Tratamiento 4: Testigo o control, esta muestra (Caja
Petri con Aspergillus) no llevó adición de fungicida natural y químico. Tratamiento 5: Aplicación del fungicida químico (Skul): Se aplicó las respectivas dosis controladas.

\section{E.Método Tukey}

El análisis funcional para las comparaciones de los tratamientos se realizó a partir de la prueba de Tukey a un nivel de confianza del $95 \%$ de probabilidad, donde el coeficiente de variación se lo expresará en (\%) [11]. Debido a que se realizaron comparaciones múltiples y considerando la tasa de error, el método Tukey se ajusta al proyecto ya que la probabilidad de cometer un error de tipo I para una serie de comparaciones es mayor que la tasa de error para cualquier comparación individual.

\section{IV.RESULTADOS}

En esta sección se presentan los resultados obtenidos a partir de la aplicación de extractos naturales de Neem (Azadirachta indico) y Eucalipto (Eucaliptus spp) en cultivos de Aspergillus spp. como proceso de inactivación fúngica.

\section{A.Extractos naturales con función antifúngica a partir Azadirachta indico y Eucaliptus spp.}

La Tabla I muestra el peso de la hoja, mililitros de agua autoclava y etanol $96 \%$ utilizado en el proceso de elaboración del fungicida.

TABLA I. Peso del material vegetal a extraer

\begin{tabular}{ccccc}
\hline Especie & Parte de la planta & Peso(g) & $\begin{array}{c}\text { Agua } \\
\text { autoclavada (ml) }\end{array}$ & Etanol 96\% (ml) \\
\hline Azadirachta indico & Hoja & $100 \mathrm{~g}$ & $500 \mathrm{ml}$ & $500 \mathrm{ml}$ \\
Eucaliptusspp & Hoja & $100 \mathrm{~g}$ & $500 \mathrm{ml}$ & $500 \mathrm{ml}$ \\
\hline
\end{tabular}

B.Efecto antifúngico en A. terreus mediante dosificación controlada de A. indico y Eucaliptus spp.

La Tabla II muestra el área de crecimiento de Aspergillus terreus durante tres días consecutivos, el día número 1 se agregaron 4 discos de papel filtro humedecidos con Neem en las cajas Petri y luego fue colocado un disco más por día. Los valores tuvieron un área de crecimiento significativo.

TABLA II. Área de crecimiento con aplicación de Neem

\begin{tabular}{ccccc}
\hline \multirow{2}{*}{$\mathbf{N}^{\mathbf{0}}$} & Medio de cultivo & \multicolumn{3}{c}{ Días } \\
\cline { 3 - 5 } & & $\mathbf{1}$ & $\mathbf{2}$ & $\mathbf{3}$ \\
\cline { 3 - 5 } 1 & Dextrosa & & $\mathbf{c m}^{\mathbf{2}}$ & \\
\hline & $\begin{array}{l}\text { Saboraud } \\
\text { Dextrosa }\end{array}$ & 0,25 & 15,82 & 32,45 \\
2 & $\begin{array}{l}\text { Saboraud } \\
\text { Dextrosa }\end{array}$ & 1 & 29,25 & 59,31 \\
3 & $\begin{array}{l}\text { Saboraud } \\
\text { Dextrosa }\end{array}$ & 0 & 11,8 & 25,5 \\
4 & $\begin{array}{l}\text { Saboraud } \\
\text { Dextrosa }\end{array}$ & 0 & 11,9 & 28,24 \\
5 & Saboraud & 0,25 & 31 & 32,3 \\
\hline
\end{tabular}


La Tabla III indica el área de crecimiento de Aspergillus terreus en cajas Petri frente a extracto de Eucalipto, se evidencia el crecimiento del hongo por día, los valores fueron un poco más bajos que el tratamiento de Neem.

TABLA III. Área de crecimiento con aplicación de Eucalipto.

\begin{tabular}{|c|c|c|c|c|}
\hline \multirow{3}{*}{$\mathbf{N}^{0}$} & \multirow{3}{*}{ Medio de cultivo } & \multicolumn{3}{|c|}{ Días } \\
\hline & & 1 & & 3 \\
\hline & & & & \\
\hline 1 & Dextrosa Saboraud & 0 & 19 & 25,56 \\
\hline 2 & Dextrosa Saboraud & 0 & 22 & 33,53 \\
\hline 3 & Dextrosa Saboraud & 1,25 & 28,5 & 36,25 \\
\hline 4 & Dextrosa Saboraud & 0 & 7 & 14,69 \\
\hline 5 & Dextrosa Saboraud & 0,25 & 22,4 & 33,38 \\
\hline
\end{tabular}

La Tabla IV muestra el área de crecimiento por día lipto, los valores indican menor crecimiento del hongo de A. terreus con aplicación de mezcla Neem + Euca- a diferencia de los tratamientos naturales 1 y 2.

TABLA IV. Área de crecimiento con aplicación de mezcla Neem + Eucalipto.

\begin{tabular}{ccccc}
\hline & & \multicolumn{3}{c}{ Días } \\
\cline { 3 - 5 } $\mathbf{N}^{\mathbf{0}}$ & Medio de cultivo & $\mathbf{1}$ & $\mathbf{2}$ & $\mathbf{3}$ \\
\cline { 3 - 5 } & & \multicolumn{3}{c}{$\mathbf{c m}^{\mathbf{2} *}$} \\
\hline 1 & Dextrosa Saboraud & 0 & 12 & 16,98 \\
2 & Dextrosa Saboraud & 0 & 15,64 & 16,9 \\
3 & Dextrosa Saboraud & 0 & 14,43 & 28,6 \\
4 & Dextrosa Saboraud & 0,34 & 16,55 & 22 \\
5 & Dextrosa Saboraud & 0 & 14,6 & 23,23 \\
\hline
\end{tabular}

La Tabla V indica el tratamiento 4, control o testigo. En este tratamiento no hubo aplicación de fungicida.

TABLA V. Área de crecimiento sin aplicación de fungicida.

\begin{tabular}{ccccc}
\hline & & \multicolumn{3}{c}{ Días } \\
\cline { 3 - 5 } $\mathbf{N}^{\mathbf{0}}$ & Medio de cultivo & $\mathbf{1}$ & $\mathbf{2}$ & $\mathbf{3}$ \\
\cline { 3 - 5 } & & & $\mathbf{c m}^{\mathbf{2}}$ & 56,1 \\
& Dextrosa Saboraud & 52,4 & 55,9 & 51,2 \\
2 & Dextrosa Saboraud & 43,8 & 48,7 & 61,7 \\
3 & Dextrosa Saboraud & 50,31 & 59,7 & 63,3 \\
4 & Dextrosa Saboraud & 61,07 & 63,3 & 63,61 \\
\hline 5 & Dextrosa Saboraud & 59,8 & 60,1 & 5 \\
\hline
\end{tabular}

En la Tabla VI se evidencia que no hubo crecimiento químico no permitió el crecimiento del hongo desde su de A. terreus, los valores fueron de cero. El fungicida primer día de aplicación.

TABLA VI. Área de crecimiento con aplicación de fungicida químico Skul.

\begin{tabular}{ccccc}
\hline & & & \multicolumn{3}{c}{ Días } \\
\cline { 3 - 5 } $\mathbf{N}^{\mathbf{2}}$ & Medio de cultivo & $\mathbf{1}$ & $\mathbf{2}$ & $\mathbf{3}$ \\
\cline { 3 - 5 } $\mathbf{c m}^{\mathbf{2} *}$ & & \multicolumn{3}{c}{0} \\
\hline 1 & Dextrosa Saboraud & 0 & 0 & 0 \\
2 & Dextrosa Saboraud & 0 & 0 & 0 \\
3 & Dextrosa Saboraud & 0 & 0 & 0 \\
4 & Dextrosa Saboraud & 0 & 0 & 0 \\
\hline
\end{tabular}


La Tabla VII que se muestra a continuación es el Análisis de varianza entre los días 1, 2, 3 frente al tratamiento de Neem. La razón -F, que en este caso es igual a 17,0794, es el cociente entre el estimado entre grupos y el estimado dentro de grupos. Puesto que el valor -P de la prueba $-\mathrm{F}$ es menor que 0,05 , existe una diferencia estadísticamente significativa entre las medias de las 3 variables con un nivel del $95,0 \%$ de confianza.

TABLA VII. Análisis ANOVA en tratamiento de Neem.

\begin{tabular}{cccccc}
\hline Fuente & $\begin{array}{c}\text { Suma de } \\
\text { Cuadrados }\end{array}$ & Gl & $\begin{array}{c}\text { Cuadrado } \\
\text { Medio }\end{array}$ & Razón-F & Valor-P \\
\hline Entre grupos & 3121,82 & 2 & 1560,91 & 17,08 & 0,0003 \\
Intra grupos & 1096,7 & 12 & 91,3915 & & \\
Total (Corr.) & 4218,52 & 14 & & & \\
\hline
\end{tabular}

La Tabla VIII muestra las medias de área de creci- Neem miento de Aspergillus terreus frente al tratamiento de

TABLA VIII. Medias con intervalos de confianza del 95,0\% en tratamiento de Neem

\begin{tabular}{cccccc}
\hline & Casos & Media & (s agrupada) & Límite Inferior & $\begin{array}{c}\text { Límite } \\
\text { Superior }\end{array}$ \\
\hline Día 1 & 5 & 0,3 & 4,27531 & $-6,28679$ & 6,88679 \\
Día 2 & 5 & 19,954 & 4,27531 & 13,3672 & 26,5408 \\
Día 3 & 5 & 35,56 & 4,27531 & 28,9732 & 42,1468 \\
Total & 15 & 18,6047 & & & \\
\hline
\end{tabular}

La Tabla IX que se muestra es el análisis de varianza aplicado al tratamiento A. terreus frente al extracto de Eucalipto. La razón-F de la Tabla IX, que en este caso es igual a 22,5231, es el cociente entre el estimado entre grupos y el estimado dentro de grupos. Puesto que el valor $-\mathrm{P}$ de la prueba $-\mathrm{F}$ es menor que 0,05 , existe una diferencia estadísticamente significativa entre las medias de las 3 variables con un nivel del 95,0\% de confianza.

TABLA IX. Análisis ANOVA en tratamiento de Eucalipto.

\begin{tabular}{cccccc}
\hline \multicolumn{1}{c}{ Fuente } & $\begin{array}{c}\text { Suma de } \\
\text { Cuadrados }\end{array}$ & $\boldsymbol{G l}$ & $\begin{array}{c}\text { Cuadrado } \\
\text { Medio }\end{array}$ & Razón-F & Valor-P \\
\hline Entre grupos & 2107,09 & 2 & 1053,54 & 22,52 & 0,0001 \\
Intra grupos & 561,315 & 12 & 46,7762 & & \\
Total (Corr.) & 2668,4 & 14 & & & \\
\hline
\end{tabular}

La Tabla X indica las medias de crecimiento de A. terreus en el tratamiento de Eucalipto

TABLA X. Medias con intervalos de confianza del 95,0\% en tratamiento de Eucalipto

\begin{tabular}{cccccc}
\hline & Casos & Media & (s agrupada) & Límite Inferior & $\begin{array}{c}\text { Límite } \\
\text { Superior }\end{array}$ \\
\hline Día 1 & 5 & 0,3 & 3,05863 & $-4,41231$ & 5,01231 \\
Día 2 & 5 & 19,78 & 3,05863 & 15,0677 & 24,4923 \\
Día 3 & 5 & 28,682 & 3,05863 & 23,9697 & 33,3943 \\
Total & 15 & 16,254 & & & \\
\hline
\end{tabular}


La Tabla XI indica el Analisis de Varianza aplicado al tratamiento de Neem + Eucalipto. La razón -F de la Tabla XI, que en este caso es igual a 67,4056 , es el cociente entre el estimado entre grupos y el estimado den- tro de grupos. Puesto que el valor-P de la prueba $-\mathrm{F}$ es menor que 0,05 , existe una diferencia estadísticamente significativa entre las medias de las 3 variables con un nivel del 95,0\% de confianza.

TABLA XI. Análisis ANOVA para Neem+Eucalipto

\begin{tabular}{cccccc}
\hline Fuente & $\begin{array}{c}\text { Suma de } \\
\text { Cuadrados }\end{array}$ & $\boldsymbol{G l}$ & $\begin{array}{c}\text { Cuadrado } \\
\text { Medio }\end{array}$ & Razón-F & Valor-P \\
\hline Entre grupos & 1201,96 & 2 & 600,979 & 67,41 & 0 \\
Intra grupos & 106,99 & 12 & 8,91586 & & \\
Total (Corr.) & 1308,95 & 14 & & & \\
\hline
\end{tabular}

La Tabla XII muestra las medias por días de creci- + Eucalipto). miento de A. terreus frente a mezcla de extractos (Neem

TABLA XII. Medias con intervalos de confianza del 95,0 para Neem + Eucalipto.

\begin{tabular}{lccccc}
\hline & Casos & Media & (s agrupada) & Límite Inferior & $\begin{array}{c}\text { Límite } \\
\text { Superior }\end{array}$ \\
\hline Día 1 & 5 & 0,068 & 1,33535 & $-1,98932$ & 2,12532 \\
Día 2 & 5 & 14,644 & 1,33535 & 12,5867 & 16,7013 \\
Día 3 & 5 & 21,542 & 1,33535 & 19,4847 & 23,5993 \\
Total & 15 & 12,0847 & & & \\
\hline
\end{tabular}

La Tabla XIII muestra el Análisis de Varianza para el tratamiento de Control o testigo donde la razón-F, que en este caso es igual a 1,16649 , es el cociente entre el estimado entre grupos y el estimado dentro-de-grupos.
Puesto que el valor-P de la razón-F es mayor o igual que 0,05 , no existe una diferencia estadísticamente significativa entre las medias de las 3 variables con un nivel del $95,0 \%$ de confianza.

TABLA XIII. Análisis ANOVA para Control o testigo

\begin{tabular}{cccccc}
\hline Fuente & $\begin{array}{c}\text { Suma de } \\
\text { Cuadrados }\end{array}$ & Gl & $\begin{array}{c}\text { Cuadrado } \\
\text { Medio }\end{array}$ & Razón-F & Valor-P \\
\hline Entre grupos & 86,2845 & 2 & 43,1422 & 1,17 & 0,3444 \\
Intra grupos & 443,817 & 12 & 36,9847 & & \\
Total (Corr.) & 530,101 & 14 & & & \\
\hline
\end{tabular}

La tabla XIV muestra las medias de crecimiento de químico. A. terreus por día sin aplicación de fungicida natural y

TABLA XIV. Medias con intervalos de confianza del 95,0 para Control o testigo

\begin{tabular}{cccccc}
\hline & Casos & Media & (s agrupada) & Límite Inferior & $\begin{array}{c}\text { Límite } \\
\text { Superior }\end{array}$ \\
\hline Día 1 & 5 & 53,476 & 2,71973 & 49,2858 & 57,6662 \\
Día 2 & 5 & 57,54 & 2,71973 & 53,3498 & 61,7302 \\
Día 3 & 5 & 59,182 & 2,71973 & 54,9918 & 63,3722 \\
Total & 15 & 56,7327 & & & \\
\hline
\end{tabular}


C.Análisis ANOVA de comparación entre tratamientos.

La Tabla XV que se muestra a continuación es el

TABLA XV. Resumen Estadístico entre tratamientos resumen estadístico entre los tratamientos naturales y el testigo control.

\begin{tabular}{|c|c|c|c|c|c|c|c|}
\hline & Recuento & Promedio & $\begin{array}{c}\text { Desviació } \\
n \\
\text { Estándar }\end{array}$ & $\begin{array}{l}\text { Coeficient } \\
\text { e de } \\
\text { Variación }\end{array}$ & Mínimo & Máximo & Rango \\
\hline Neem & 3 & 18,6047 & 17,6687 & $94,97 \%$ & 0,3 & 35,56 & 35,26 \\
\hline Eucalipto & 3 & 16,254 & 14,5158 & $89,31 \%$ & 0,3 & 28,682 & 28,382 \\
\hline Mezcla & 3 & 12,0847 & 10,9634 & $90,72 \%$ & 0,068 & 21,542 & 21,474 \\
\hline Testigo & 3 & 56,7327 & 2,93742 & $5,18 \%$ & 53,476 & 59,182 & 5,706 \\
\hline Total & 12 & 25,919 & 21,6727 & $83,62 \%$ & 0,068 & 59,182 & 59,114 \\
\hline
\end{tabular}

La Tabla XVI muestra la razón-F, que en este caso es igual a 7,90395, es el cociente entre el estimado entre grupos y el estimado dentro de grupos. Puesto que el valor $-\mathrm{P}$ de la prueba $-\mathrm{F}$ es menor que 0,05 , existe una diferencia estadísticamente significativa entre las medias de las 4 variables con un nivel del 95,0\% de confianza por lo que se rechaza la hipótesis nula y se acepta la hipótesis alternativa.

TABLA XVI. Análisis ANOVA entre tratamientos

\begin{tabular}{cccccc}
\hline Fuente & $\begin{array}{c}\text { Suma de } \\
\text { Cuadrados }\end{array}$ & Gl & $\begin{array}{c}\text { Cuadrado } \\
\text { Medio }\end{array}$ & Razón-F & Valor- $\boldsymbol{P}$ \\
\hline Entre grupos & 3863,35 & 3 & 1287,78 & 7,9 & 0,0089 \\
Intra grupos & 1303,43 & 8 & 162,929 & & \\
Total (Corr.) & 5166,78 & 11 & & & \\
\hline
\end{tabular}

la Tabla XVII muestra las medias de crecimiento en to de A. terreus. cada uno de los tratamientos aplicados en el crecimien-

TABLA XVII. Medias con intervalos de confianza al $96,0 \%$ entre tratamientos

\begin{tabular}{lccccc}
\hline & Casos & Media & (s agrupada) & Límite Inferior & $\begin{array}{c}\text { Límite } \\
\text { Superior }\end{array}$ \\
\hline Neem & 3 & 18,6047 & 7,36951 & 6,58798 & 30,6213 \\
Eucalipto & 3 & 16,254 & 7,36951 & 4,23732 & 28,2707 \\
Mezcla & 3 & 12,0847 & 7,36951 & 0,0679839 & 24,1013 \\
Testigo & 3 & 56,7327 & 7,36951 & 44,716 & 68,7493 \\
Total & 12 & 25,919 & & & \\
\hline
\end{tabular}

La Tabla XVIII muestra los porcentajes de efectivi- dad de los tratamientos.

TABLA XVIII. Efectividad de los tratamientos

\begin{tabular}{cc}
\hline TRATAMIENTOS & EFECTIVIDAD \\
\hline Neem & $67,19 \%$ \\
Eucalipto & $71,3 \%$ \\
Mezcla & $78,6 \%$ \\
Químico & $100 \%$ \\
\hline
\end{tabular}


Estos tratamientos son eficientes debido a sus principios activos presentes en las hojas. En el caso del Eucalipto este contiene aceites esenciales como eucaliptol, monoterpenos, sesquiterpenos, aldehídos y cetonas. Aceites que tienen capacidad antimicrobiana, antibacteriana, depurativa, antiviral entre otros [12]. El Neem presenta algunos metabolitos como son nimbin, nimbidinim, azadiractina, alanina. En el caso del nimbidinim este inhibe el crecimiento de hongos [13].

Los resultados obtenidos a través de ANOVA muestran que existen diferencias significativas entre los tratamientos, por lo que fue utilizada la prueba Tukey. La Tabla XIX mostró diferencias entre el Control/Testigo, el cual obtuvo un crecimiento de $53.73 \mathrm{~cm}^{2}$ a diferencia de los tratamientos de Neem $\left(18.6 \mathrm{~cm}^{2}\right)$, Eucalipto $\left(16.25 \mathrm{~cm}^{2}\right)$ y mezcla $\left(12.08 \mathrm{~cm}^{2}\right)$.

\section{TABLA XIX. Método Tukey}

\begin{tabular}{|c|c|c|c|c|}
\hline \multicolumn{5}{|c|}{ Eucalipt } \\
\hline & Neem & 0 & Mezcla & Testigo \\
\hline Neem & & 2,3507 & 6,52 & $-38,128$ \\
\hline Eucalipt & & & & \\
\hline o & & & 4,1693 & $-40,4787$ \\
\hline Mezcla & & & & $-44,648$ \\
\hline Testigo & & & & \\
\hline
\end{tabular}

\section{V.CONCLUSIONES}

Se implementó un proceso de inactivación fúngica mediante la aplicación de extractos naturales de Azaridachta indico y Eucaliptus en cultivos de Aspergillus terreus. Los resultados de esta investigación permiten aplicar inhibidores naturales en cultivos con problemas fitopatógenos de Aspergillus, siendo estos extractos una alternativa agroecológica.

Se recolectaron plantas de Neem y Eucalipto, para realizar el extracto y demostrar la actividad antifúngica frente a Aspergillus. La inhibición en el crecimiento de A. terreus con Eucalyptus y A.indico, no es igual al fungicida de mercado Skul teniendo este un $100 \%$ de eficiencia. Sin embargo, la mezcla de extractos de Neem + Eucalipto presentan mayor inhibición con un porcentaje de eficiencia de $78.6 \%$, a estos datos le sigue el extracto de Eucalipto con un $71.3 \%$ y por último el extracto de Neem con $67.1 \%$. Aspergillus terreus crece de manera acelerada en altas temperaturas, y es capaz de crecer en temperaturas bajas. Al evaluar la efectividad antifúngica de distintos extractos vegetales frente a fungicidas de mercado mediante información bibliográfica se constató, que la mayor parte de estos presenta el porcentaje más alto de inhibición, aunque los extractos naturales tienen resultados favorables.

\section{REFERENCIAS}

[1]R. Riccioppo, «Agroquímicos,» 2011. [En línea]. Available: http://www.colmed7.org.ar/files/Trabajos/ AGROQUIMICOS.pdf.

[2]Ministerio de Salud, Secretaría de Ambiente y Desa- rrollo Sustentable, «La problemática de los agroquímicos y sus envases, su incidencia en la salud.,» Gráfica Laf S.R.L, Buenos Aires, 2007.

[3]FAO, «El estado mundial de la agricultura y la alimentación,» 2016. [En línea]. Available: http://www. fao.org/3/a-i6030s.pdf.

[4]FAO, «Anuario Estadistico de la FAO,» La Alimentacion y la Agricultura en America Latina y El Caribe, p. p10, 2014.

[5]FAO, «Anuario Estadistico de la FAO,» La Alimentacion y la Agricultura en Ameria Latina y el Caribe, pp. p134-135, 2014.

[6]INEC, «Encuesta sobre Uso de Agroquímicos y su Destino Final en la Agricultura,» 2012. [En línea]. Available: http://www.ecuadorencifras.gob.ec/wp-content/descargas/Presentaciones/Plaguicidas.pdf.

[7]INEC, «Encuesta de Superficie y Producción Agropecuaria Continua,» 2016. [En línea]. Available: http:// www.ecuadorencifras.gob.ec/documentos/web-inec/ Encuestas_Ambientales/Informacion_ambiental_en la_agricultura/2016/PRESENTACION_AGRO_AMBIENTE_2016.pdf.

[8]E1 Universo , «Agricultores, en riesgo por el uso de los agroquímicos,» Domingo Mayo 2015. [En línea]. Available: https://www.eluniverso.com/noticias/2015/05/10/nota/4853501/agricultores-riesgo-uso-agroquimicos.

[9]El Telégrafo, «Mal uso de agroquímicos afecta a la salud y el medio ambiente,» Economía, 04 Febrero 2012.

[10]V. Aguirre, M. Anrango, V. Delgado y N. Díaz, 
«Centro de Investigaciones CientÍficas ESPE,» 22 Noviembre 2012. [En línea]. Available: https://repositorio.espe.edu.ec/bitstream/21000/6845/1/AC-B-ESPE-034935.pdf.

[11]Minitab, «Método de Tukey para Comparaciones Múltiples,» 2017. [En línea]. Available: https:// support.minitab.com/es-mx/minitab/18/help-and-howto/modeling-statistics/anova/supporting-topics/multiple-comparisons/what-is-tukey-s-method/.
[12]J. Cruz, «Eucalipto,» 2007. [En línea]. Available:http://www.agaetespacioweb.com/EUCALIPTO $\% 20$ TEXTO\%20Y\%20FOTOGRAF\%C3\%8DA\%20 COMPUESTO-OK.pdf.

[13]C. Estrella, «Impregnación de aceite de Neem en soporte textil para combatir la mosca de la fruta Anastrepha striata,» Febrero 2017. [En línea]. Available: http://bibdigital.epn.edu.ec/bitstream/15000/17404/1/ CD-7905.pdf. 